\title{
Mediastinal cryptococcosis masquerading as therapy-refractory lymphoma
}

\author{
Matthew Wong • Florence Loong • Pek-Lan Khong • \\ Yok-Lam Kwong • Anskar Y. H. Leung
}

Received: 2 August 2010 /Accepted: 8 August 2010 /Published online: 21 August 2010

(C) The Author(s) 2010. This article is published with open access at Springerlink.com

\section{Dear Editor,}

A 58-year-old woman presented with a 1-month history of abdominal pain and weight loss. Positron emission tomography computerized tomography (PET-CT) showed markedly thickened and hypermetabolic small bowel (standardized uptake value maximum, SUVmax: 8.7) associated with multiple abdominal and pelvic lymphadenopathy (Fig. 1a). Intestinal obstruction soon developed, necessitating small bowel resection. Histologic examination showed enteropathy-associated T-cell lymphoma. She was treated with three cycles of chemotherapy comprising steroid, methotrexate, ifosphamide, L-asparaginase, and etoposide [1]. Interim reassessment PET-CT showed complete resolution of the abdominal and pelvic lesions (Fig. 1b). However, new pretracheal, precarinal, subcarinal, and left hilar lymphadenopathy (SUVmax, 7.4; Fig. 1b, arrows) developed. The patient was afebrile with no respiratory symptoms. Owing to discrepant responses of lymphadenopathy to chemotherapy, further diagnostic evaluation was pursued. To avoid general anesthesia necessary for mediastinoscopy, endobronchial ultrasound guided transbronchial needle aspiration (EBUSTBNA) was performed under local anesthesia with conscious sedation. Multiple mediastinal lymph nodes located in the

M. Wong $\cdot$ Y.-L. Kwong $(\bowtie) \cdot$ A. Y. H. Leung

Department of Medicine, Queen Mary Hospital,

Pokfulam Road,

Hong Kong, China

e-mail: ylkwong@hkucc.hku.hk

F. Loong

Department of Pathology, Queen Mary Hospital,

Pokfulam Road,

Hong Kong, China

P.-L. Khong

Department of Diagnostic Radiology, Queen Mary Hospital,

Pokfulam Road,

Hong Kong, China upper, lower paratracheal, and subcarinal area were aspirated. Conventional fiberoptic bronchoscopy was also performed with no endobronchial abnormality detected. Both cytologic and histologic specimens obtained from all sampled lymph nodes demonstrated epithelioid-like cells and multinucleated giant cells, with no atypical lymphoid cells found. Roundish spore-like organisms were indentified (Fig. 2a, arrows). These organisms were positive for the fungal mucicarmine (Fig. 2b, arrows) and Grocott stains. The histopathologic features were consistent with cryptococcal lymphadenitis. Serum cryptococcal antigen was also positive at 1:16. The cerebrospinal fluid was normal. She was treated with oral fluconazole (400 mg daily). A PET-CT scan after 6 weeks of fluconazole showed that the mediastinal lymphadenopathy had largely resolved.

Cryptococcal infection usually results from inhalation of fungal spores, and may be confined to the lungs or disseminate systemically. Radiologically, pulmonary cryptococcosis may present as a single well-defined consolidation or mass, diffusely scattered nodules or reticulonodules [2]. Cryptococcal lymphadenopathy, especially affecting the mediastinum, is infrequent and mainly reported in patients infected with the human immunodeficiency virus (HIV) [3, 4]. Radiologically, in HIV-negative patients, pulmonary cryptococcal infection usually presents as large nodular lesions or alveolar infiltrates. In HIV-infected patients, lymphadenopathy and interstitial infiltrates are instead the common findings [5]. In cryptococcal lymphadenitis, lymph nodes are generally $<1.5 \mathrm{~cm}$ and accompanied by pulmonary parenchymal changes [6]. Hence, the extensive multifocal mediastinal lymphadenopathy without parenchymal lesions in our patient was more consistent radiologically with lymphoma progression. However, suitable investigations finally revealed the correct diagnosis.

Conventional transbronchial needle aspiration with a histology needle in immunocompromised patients with 




Fig. 1 Positron emission tomography. a Before treatment, showing massive bowel and pelvic involvement (arrows). b After chemotherapy, showing resolution of all previous lesions, but matted mediastinal lymphadenopathy of similar metabolic activity as the original lymphoma

Fig. 2 Lymph node findings. a Histologic examination showed numerous spore-like organisms (arrows). b The organisms were positive for the fungal stain mucicarmine (arrows)
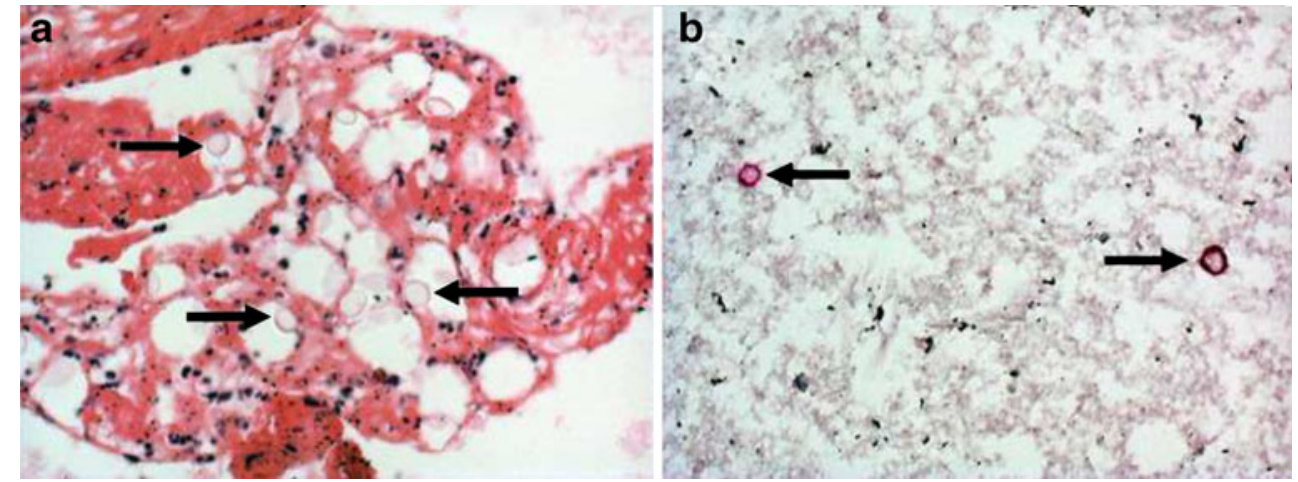

intrathoracic lymphadenopathy has a poor diagnostic yield [7]. EBUS-TBNA with a designated 22-gauge needle has significant advantages. In addition to a high diagnostic yield for mediastinal lymphadenopathy, both histologic and cytologic specimens can be obtained. A histological specimen aids in the diagnosis of non-malignant mediastinal lymphadenopathy such as sarcoidosis, as well as eliminates the possibility of false-positive microbiologic results from contamination by airway colonization. Our case illustrated well the utility of EBUS-TBNA as a minimally invasive technique in clinching the diagnosis.

Open Access This article is distributed under the terms of the Creative Commons Attribution Noncommercial License which permits any noncommercial use, distribution, and reproduction in any medium, provided the original author(s) and source are credited.

\section{References}

1. Yamaguchi M, Suzuki R, Kwong YL, Kim WS, Hasegawa Y, Izutsu K, Suzumiya J, Okamura T, Nakamura S, Kawa K,
Oshimi K (2008) Phase I study of dexamethasone, methotrexate, ifosfamide, L-asparaginase, and etoposide (SMILE) chemotherapy for advanced-stage, relapsed or refractory extranodal natural killer (NK)/T-cell lymphoma and leukemia. Cancer Sci 99:10161020

2. Fraser RG, Pare JA, Pare PD, Fraser RS, Genereux GP (1989) Diagnosis of diseases of the chest, 3rd edn. Saunders, Philadelphia, pp 975-985

3. Witt D, McKay D, Schwam L, Goldstein D, Gold J (1987) Acquired immune deficiency syndrome presenting as bone marrow and mediastinal cryptococcosis. Am J Med 82:149150

4. Wannakrairot P, Leong TY, Leong AS (2007) The morphological spectrum of lymphadenopathy in HIV infected patients. Pathology 39:223-227

5. Miller WT, Edelman JM, Miller WT (1990) Cryptococcal pulmonary infection in patients with AIDS: radiographic appearance. Radiology 175:725-728

6. Lacomis JM, Costello P, Vilchez R, Kusne S (2001) The radiology of pulmonary cryptococcosis in a tertiary medical center. J Thorac Imaging 16:139-148

7. Harkin TJ, Ciotoli C, Addrizzo-Harris DJ, Naidich DP, Jagirdar J, Rom WN (1998) Transbronchial needle aspiration (TBNA) in patients infected with HIV. Am J Respir Crit Care Med 157:19131918 